extracolonic findings. Extracolonic abnormalities were found in 383 patients (46\%). Of those patients with extracolonic findings $9 \%$ had extracolonic malignancies, $26 \%$ had important extracolonic findings requiring further investigation, management or referral and $65 \%$ were benign incidental findings requiring no further follow-up. The most common benign incidental finding was renal cysts and the most common extracolonic malignancy was renal carcinoma. Abstract OC-152 table 1 gives a summary of the number of extracolonic findings identified with the highest prevalence.

Abstract 0C-152 Table 1 Number of Extracolonic Findings by Category

\begin{tabular}{lrlrll}
\hline Benign incidental & & Benign important & & Malignancy & \\
\hline Renal cyst & 86 & Adrenal mass $>2 \mathrm{~cm}$ & 10 & Bladder & 2 \\
Hepatic cyst/granuloma & 68 & Complex liver lesion & 4 & Endometrial & 2 \\
Adrenal mass & 12 & Complex pancreatic lesion & 2 & Gastric & 2 \\
Pancreatic cyst & 11 & Adnexal mass & 28 & Lung & 2 \\
AAA $<5 \mathrm{~cm}$ & 14 & AAA $>5 \mathrm{~cm}$ & 10 & Myeloma & 1 \\
Renal calculi & 8 & Upper Gl lymphadenopathy & 8 & Ovarian & 4 \\
Hernia & 22 & & & Pancreatic & 5 \\
Hiatus hernia & 41 & & Renal & 7 \\
& & & Unknown primary & 2 \\
\hline
\end{tabular}

Conclusion CT Colonography has the potential to pick up cancers and other life threatening lesions such as large non ruptured AAA at a preclinical stage. While we acknowledge that extracolonic abnormalities are common with the correct planning and management we do not believe that this should necessarily increase the number of further unnecessary investigations or costs.

Competing interests None declared.

\section{OC-153 ULTRASOUND-DETECTED GALLBLADDER POLYPOID LESIONS, WHAT IS NEXT?}

doi:10.1136/gutjnl-2012-302514a.153

A Kambal, ${ }^{*}$ C Brown, R Kannan, 0 Jalil, A Feroz, A Rasheed. General Surgery, Gwent institute for minimal access surgery, Newport, UK

Introduction To determine the nature and assess the current clinical pathways for the ultrasound-detected gallbladder polyps (GBP) and propose a follow-up and a therapeutic strategy based on size and symptomatology.

Methods A retrospective search of the US database for "polyp" in gallbladder for the period between 1st January 2009 and 31st December 2010 was conducted. A database was then constructed including demographics, clinical presentation, principal symptoms, management and pathology. Histologic findings were analysed in patients who underwent cholecystectomy. The electronic medical records were searched to check the clinical outcome, pathologic data and follow-up arrangements.

Results Ultrasound detected gallbladder polyps were reported in 347 patients, 214 female and 133 males with an age range between 14 and 93 yrs, (Median 5, IOR=41-58). Polyps were found during the course of investigation for the possibility of gallbladder disease in 125 patients (36\%). The rest were incidental finding during investigation of other illnesses. The majority of referrals for the US came from primary care $(60 \%)$, the rest came from hospital physicians in $(24 \%)$ and surgeons in $(13 \%)$ of cases. Forty two percent of the reports made no mention of polyp actual size and 39\% reported the polypoid lesion to be

Conclusion Our preliminary data confirm that the majority of the US detected "polypoid lesions" are incidental findings and are not true epithelial polyps. The majority of patients with symptomatic polyps who underwent cholecystectomy had cholelithiasis on histology. The current management strategy of asymptomatic polyps relies on its size and hence must be included in the US report which was poorly complied with in this series. Small $(10 \mathrm{~mm}$ should be offered cholecystectomy. A well defined long term follow-up of patients is necessary to allow better understanding of this pathological entity and a nationwide registry or large longitudinal observational study is warranted as these might represent a missed opportunity for early detection of cancer.

Competing interests None declared.

\section{Gastrointestinal physiology associates group (AGIP) symposium: "reflux associated cough" OC-154 INTER-OBSERVER AGREEMENT FOR MANOMETRY CLASSIFICATION OF INDIVIDUAL SWALLOWS AND DIAGNOSES USING HIGH-RESOLUTION MANOMETRY (HRM) WITH ESOPHAGEAL PRESSURE TOPOGRAPHY (EPT): RESULTS OF HIGH PARTICIPATION WEB-BASED STUDIES BY THE HRM WORKING GROUP}

doi:10.1136/gutjnl-2012-302514a.154

${ }^{1} \mathrm{M}$ R Fox, ${ }^{*}$ J Pandolfino, ${ }^{3} \mathrm{~J}$ Jafari, ${ }^{4} \mathrm{D}$ Menne. ${ }^{1} \mathrm{~N} / \mathrm{HR}$ Biomedical Research Unit, Nottingham Digestive Diseases Centre, Nottingham, UK; ${ }^{2}$ Feinberg School of Medicine, NW University, Chicago, USA; ${ }^{3}$ The Wingate Institute, Digestive Diseases, Barts and The London School of Medicine, London, UK; ${ }^{4}$ Biostatistics, Menne Biomed, Tuebingen, Germany

Introduction Recently the HRM Working Group presented a classification system for esophageal motility disorders using HRM/EPT (Pandolfino, NGM 2009). This study reports inter-observer agreement for classification of individual HRM/EPT water swallows (study A) and diagnoses based on 10 swallows (study B) using this system.

Methods All registered members of the hrmconsensus.org website were invited to take part. Institution and experience with HRM were recorded. A sample of 147 individual swallows (study A) and 40 diagnostic studies (study B) was reviewed and classified using a drop down menu that utilised the published system. The on-line platform provided a generic EPT format with fixed pressure scale and time base. Contours facilitated analysis of intra-bolus and contractile pressure; however no data summary was provided. The sequence of swallows was fixed for each user, but randomised between users to avoid sequence bias. Users were blinded to other participant's entries. During the study users were free to reclassify and post comments; however, once completed classifications could not be changed.

Results (A) All 147 individual swallows were assessed by 18 users High levels of agreement ( $\leq 2 / 18$ dissenters) were present for normal peristalsis and achalasia but lower levels for peristaltic and intrabolus pressure (IBP) abnormalities. (B) All 40 diagnostic studies were assessed by 36 users. Overall inter-observer agreement was fair $(\kappa$ $0.42)$ being higher $(\kappa>0.5)$ for aperistalsis and achalasia, and lower $(\kappa<0.4)$ for peristaltic abnormalities. Users with $>400 \mathrm{HRM} / \mathrm{EPT}$ studies showed somewhat better agreement $(n=9 ; \kappa 0.46)$ and agreement was good for users in the US institution that developed the classification system $(n=4 ; \kappa \quad 0.57)$. Analysis of comments revealed that disagreements in both studies were not random but due to (1) multiple abnormalities in single swallows (2) limited functionality of on-line software/lack of a data summary that confounded user's ability to distinguish closely related diagnoses, especially those that depend on precise pressure measurement.

Conclusion This is the largest assessment of inter-observer agreement performed for manometric studies. Overall inter-observer agreement for HRM/EPT of individual swallows and diagnostic studies was moderate, increasing with experience of this technology and the classification system. Analysis of swallows with low 
agreement indicate that "pattern recognition" of HRM/EPT was not adequate and highlighted the value of objective metrics in diagnosis of esophageal dysmotility.

Competing interests $M$ Fox consultant for: given imaging, paid instructor for: Sandhill MMS, J Pandolfino: None declared, J Jafari: None declared, D Menne: None declared.

\section{OC-155 NO WAY BACK-IRREVOCABLE ALTERATION OF THE GASTRIC AND OESOPHAGEAL MICRO-ENVIRONMENT FOLLOWING CHOLECYSTECTOMY}

doi:10.1136/gutjnl-2012-302514a.155

S N S Gilani, ${ }^{*}$ G Bass, T N Walsh. RCSI Academic Department of Surgery, Connolly Hospital, Dublin, Ireland

Introduction Background: Loss of the gallbladder reservoir function at cholecystectomy may critically alter the dynamics of bile storage and release. Consequent iatrogenic duodeno-gastro-esophageal reflux (DGER) may be associated with oesophago-gastric adenocarcinoma. Aims To examine the histological and molecular effects of cholecystectomy-induced DGER on gastric and oesophageal mucosae.

Methods Patients and Methods: In a retrospective study we compared 26 gallstone-free controls with 25 patients pre-cholecystectomy and 29 patients post-cholecystectomy for one or more years. In a prospective study we compared 26 controls with 25 patients before and within 1 year of cholecystectomy. All underwent oesophago-gastro-duodenoscopy (EGD) with biopsies from the antrum, esophagogastric junction (EGJ) and $5 \mathrm{~cm}$ above the EGJ. A histochemical bile reflux index (BRI) was calculated and immunohistochemistry was performed for p53 and Ki67.

Results Results: In the retrospective study antral BRI positivity was $11 \%$ in controls vs $69 \%$ in cholecystectomy patients ( $p=0.001$ ); at the EGJ BRI positivity was $19 \%$ in controls vs $41 \%$ in cholecystectomy patients $(\mathrm{p}=0.032)$. $\mathrm{p} 53$ was expressed at the antrum in $4 \%$ of controls vs $52 \%$ cholecystectomy patients $(\mathrm{p}=0.001)$ and in $19 \%$ vs $66 \%$ at the EGJ ( $p=0.001)$. Ki67 was expressed at the antrum in $23 \%$ vs $59 \%(p=0.001)$ and at the EGJ in $19 \%$ vs $62 \%(p=0.001)$. Prospectively, BRI positivity increased from $11 \%$ to $36 \%(p=0.04)$ at the antrum within 1 year of cholecystectomy. Ki67 expression increased from $19 \%$ to $48 \%(p=0.044)$ at EGJ in patients within 1 year of cholecystectomy while p53 remained unchanged.

Conclusion Changes attributable to DGER occur early in the gastric and LES mucosae of patients following cholecystectomy. Ki67 and p53 over-expression suggests that these changes may be precursors of malignant transformation. Such concerning changes suggest that options other than cholecystectomy be considered for patients with gallstones in a functioning gallbladder.

Competing interests None declared.

\section{DDF symposium: "bowel cancer screening"}

\section{OC-156 ANALYSIS OF COLORECTAL POLYPS DETECTED IN THE ENGLISH NHS BOWEL CANCER SCREENING PROGRAMME WITH EMPHASIS ON ADVANCED ADENOMA AND POLYP CANCER DETECTED}

doi:10.1136/gutjnl-2012-302514a.156

${ }^{1} \mathrm{D}$ Majumdar, ${ }^{*}{ }^{2} \mathrm{~J}$ Patnick, ${ }^{2} \mathrm{C}$ Nickerson, ${ }^{1} \mathrm{M} \mathrm{D}$ Rutter. ${ }^{1}$ Department of Gastroenterology, University Hospital of North Tees, Stockton on Tees, UK; ${ }^{2}$ NHS Cancer Screening Programmes, NHS Cancer Screening Programmes, Sheffield, UK

Introduction Colorectal cancer is the second most common cause of cancer related death in the UK causing around 16000 deaths each year. Colorectal adenomas are slow growing precursor lesions which progress to cancer. The lesion of most interest in this context is advanced adenoma (size $10+\mathrm{mm} /$ with $20 \%-25 \%$ villous histology/ high grade dysplasia) as they are of higher risk of progression (2). This study analysed adenomatous lesions detected in NHS BCSP programme.

Methods Data on each patient entering the NHS BCSP programme is prospectively recorded on the national BCSP database. The database was interrogated for all polyps/adenomas found during the period September 2006 to September 2011. The data were analysed with particular focus on detection of advanced adenoma and polyp cancers. Results A total of 65535 polyps were found, of which 43954 $(67.06 \%)$ were confirmed histologically as adenomas. 15261 advanced adenomas were detected. These accounts for $34.7 \%$ of lesions removed and $23.9 \%$ of all lesions detected during screening. 842 polyp cancers were found and removed. $1.9 \%$ of the adenomatous lesions removed were polyp cancer. The incidence of villous morphology, HGD and polyp cancer, categorised by adenoma size, are shown in the Abstract OC-156 table 1. The presence of villous histology and high grade dysplasia increases with increasing size of adenoma, whereas villous histology begins to plateau for adenomas over $15 \mathrm{~mm}$ in size, the incidence of HGD appears linear up to and beyond adenomas of $45 \mathrm{~mm}$ in size.

Abstract OC-156 Table 1 Advanced histological feature and cancer in different size groups of polyp

\begin{tabular}{llllllll}
\hline & $\begin{array}{l}\text { Total } \\
\text { number } \\
\text { of adenoma }\end{array}$ & $\begin{array}{l}\text { Polyp } \\
\text { cancers }\end{array}$ & $\begin{array}{l}\% \text { Of polyp } \\
\text { cancer }\end{array}$ & HGD & $\begin{array}{l}\text { \% Of } \\
\text { HGD }\end{array}$ & $\begin{array}{l}\text { Villous } \\
\text { histology }\end{array}$ & $\begin{array}{l}\text { \% Of villous } \\
\text { histology }\end{array}$ \\
\hline & 18533 & 31 & 0.16 & 152 & 0.8 & 1035 & 5.5 \\
$5-9 \mathrm{~mm}$ & 9577 & 48 & 0.5 & 299 & 3.1 & 1781 & 18.6 \\
$10-14 \mathrm{~mm}$ & 5159 & 193 & 3.7 & 609 & 11.8 & 2381 & 46.1 \\
$15-19 \mathrm{~mm}$ & 3055 & 181 & 5.9 & 567 & 18.5 & 1904 & 62.3 \\
$20-24 \mathrm{~mm}$ & 1540 & 102 & 6.6 & 408 & 26.4 & 1048 & 68.0 \\
$25-29 \mathrm{~mm}$ & 683 & 70 & 10.2 & 211 & 30.9 & 511 & 74.8 \\
$30-34 \mathrm{~mm}$ & 401 & 48 & 11.9 & 139 & 34.6 & 299 & 74.5 \\
$35-39 \mathrm{~mm}$ & 115 & 21 & 20 & 42 & 36.5 & 93 & 80.8 \\
$40-44 \mathrm{~mm}$ & 138 & 20 & 14.4 & 54 & 39.5 & 106 & 76.9 \\
\hline
\end{tabular}

Conclusion $67.06 \%$ of all lesions found were histologically confirmed colorectal adenomas. One third of adenomas were advanced adenomas. There is a trend of increase of incidence of cancer and features of advanced neoplasia in adenomas with increasing size. The incidence of AA feature present in lesions below $10 \mathrm{~mm}$ in size was $10.07 \%$.

Competing interests None declared.

\section{REFERENCES}

Office of National Statistics. UK, 2010.

2. Atkin W, Marson B, Cuzick J. Long term risk of colorectal cancer after excision of ectosigmoid adenomas. N Engl J Med 1992;326:658-62.

\section{BSG symposium: "stem cells" \\ OC-157 PHYSIOLOGICAL CHANGES IN MATRIX STIFFNESS MODULATE HEPATIC PROGENITOR CELL MORPHOLOGY, PROLIFERATION AND DIFFERENTIATION}

doi:10.1136/gutjnl-2012-302514a.157

${ }^{1} \mathrm{~T}$ T Gordon-Walker, ${ }^{2}{ }^{2} \mathrm{~J}$ Schrader, ${ }^{1} \mathrm{~L}$ Boulter, ${ }^{1} \mathrm{~A} J \mathrm{~J}$ Robson, ${ }^{1} \mathrm{~S} J$ J Forbes, ${ }^{3,4} \mathrm{R}$ G Wells, ${ }^{1} \mathrm{~J}$ P Iredale. ${ }^{1}$ MRC Centre for Inflammation Research, Queen's Medical Research Institute, Edinburgh, UK; ${ }^{2}$ Department of Medicine I, University Medical Center Hamburg-Eppendorf, Hamburg, Germany; ${ }^{3}$ Department of Medicine, School of Medicine, University of Pennsylvania, Philadelphia, USA; ${ }^{4}$ Department of Gastroenterology, School of Medicine, University of Pennsylvania, Philadelphia, USA

Introduction Liver injury is associated with changes in the biochemical and physical properties of the extracellular matrix 